\title{
Electron Kinetic Effects in the Nonlinear Evolution of a Driven Ion-Acoustic Wave
}

\author{
C. Riconda, ${ }^{1,2}$ A. Heron, ${ }^{1}$ D. Pesme,${ }^{1}$ S. Hüller, ${ }^{1}$ V. T. Tikhonchuk, ${ }^{2}$ and F. Detering ${ }^{1}$ \\ ${ }^{1}$ Centre de Physique Théorique, École Polytechnique, 91128 Palaiseau Cedex, France \\ ${ }^{2}$ CELIA, UMR 5107 CNRS-Université Bordeaux 1-CEA, 33405 Talence Cedex, France
}

(Received 16 February 2004; published 9 February 2005)

\begin{abstract}
The electron kinetic effects are shown to play an important role in the nonlinear evolution of a driven ion-acoustic wave. The numerical simulation results obtained (i) with a hybrid code, in which the electrons behave as a fluid and the ions are described along the particle-in-cell (PIC) method, are compared with those obtained (ii) with a full-PIC code, in which the kinetic effects on both species are retained. The electron kinetic effects interplay with the usual fluid-type nonlinearity to give rise to a broadband spectrum of ion-acoustic waves saturated at a low level, even in the case of a strong excitation. This low asymptotic level might solve the long-standing problem of the small stimulated Brillouin scattering reflectivity observed in laser-plasma interaction experiments.
\end{abstract}

DOI: 10.1103/PhysRevLett.94.055003

The control of the stimulated Brillouin scattering (SBS) instability remains one of the key issues for the success of laser fusion. In the context of laser-plasma interaction, stimulated Brillouin scattering is the process by which an incident laser wave couples to an ion-acoustic wave (IAW) to give rise to a scattered transverse wave, leading to a net energy loss in the case of backscattering. The energy loss is measured by the backward SBS reflectivity, which is the ratio of the backscattered energy on the laser beam energy. One of the pending questions concerning laser-plasma interaction is the discrepancy between the experimentally observed reflectivity, $R_{\exp }$, and the theoretically predicted reflectivity, $R_{\text {pred }}$. For typical plasma parameters corresponding to present day laser-plasma experiments, the predictions based on a linear behavior of the ion-acoustic wave lead to $R_{\text {pred }}$ close to unity, when $R_{\text {exp }}$ does not exceed a few percent [1]. Since the SBS reflectivity is directly proportional to the IAW amplitude, it is therefore recognized that the SBS driven ion-acoustic wave behaves in a nonlinear way, saturating at a very low fluctuation level, consistently with the experimental results.

Various nonlinear mechanisms have been invoked to explain the IAW saturation, including fluid-type effects [2-5] and ion kinetic effects [6-10]. Accounting for fluid-type effects leads to a chaotic SBS behavior, resulting in a time averaged SBS reflectivity $\left\langle R_{\text {pred }}\right\rangle$ which is reduced in comparison with the linear IAW prediction $[3,11]$. Nonetheless, the fluid nonlinearities alone are not sufficient to explain the low reflectivities observed experimentally in long scale length plasmas. This discrepancy is one of the reasons for the renewed interest in modeling kinetic effects in the SBS studies. Most of the numerical simulations of SBS have been carried out so far with hybrid codes, in which the ions are dealt with along the particlein-cell (PIC) method and the electrons are considered to behave as an isothermal Boltzmann fluid $[4,6,8,12]$.

In this Letter, we compare the results provided by a hybrid code with those obtained with a full-PIC code, in
PACS numbers: 52.35.Fp, 52.35.Mw, 52.65.Rr

which the kinetic effects on both species are described. We find that the conventional hypothesis of isothermal Boltzmann electrons is inadequate. The main result of our Letter is, indeed, that the electron kinetic effects facilitate the ion-acoustic wave instabilities, resulting asymptotically in a broadband spectrum of IAW waves that saturate at a very low level. A second conclusion concerns the possibility of modeling the kinetic effects in fluid-type equations by means of a nonlinear frequency shift. Our full-PIC numerical results show that the electron kinetic effects cannot be reduced to a single nonlinear frequency shift.

For simplicity, we restrict ourselves to one-dimensional simulations. An IAW is driven in a homogeneous plasma by an externally imposed potential, representing the ponderomotive potential in the case of SBS, of the form $\phi_{p}=\phi_{d} \sin \left(k_{m} z-\omega_{m} t\right) ; \omega_{m}=\omega_{L}\left(k_{m}\right) \equiv c_{s e} k_{m}[1 /(1+$ $\left.\left.k_{m}^{2} \lambda_{D e}^{2}\right)+3 T_{i} / Z T_{e}\right]^{1 / 2}$ and $k_{m}$ satisfy the linear IAW dispersion relation. Here $c_{s e} \equiv\left(Z T_{e} / m_{i}\right)^{1 / 2}, T_{e}$ and $T_{i}$ are the electron and ion temperatures, respectively, $Z e$ and $m_{i}$ are the ion charge and mass; $\lambda_{\mathrm{De}} \equiv\left(\epsilon_{0} T_{e} / e^{2} n_{e 0}\right)^{1 / 2}$ denotes the Debye length, where $n_{e 0}$ is the electron background density. In the full-PIC simulations, the force acting on the electrons is $e \nabla\left(\phi+\phi_{p}\right)$, where $\phi$ denotes the selfconsistent electrostatic potential. In the hybrid simulations, the electron density is given by $n_{e}(\phi)=n_{e 0} \exp [e(\phi+$ $\left.\left.\phi_{p}\right) / T_{e}\right]$. We chose $\left(k_{m} \lambda_{\text {De }}\right)^{2}=0.1, Z=1, T_{i} / T_{e}=0.09$, leading to the linear Landau ion damping value $\nu_{i}^{L} \simeq$ $0.07 \omega_{m}$; the ion to electron mass ratio was $m_{i} / m_{e}=$ 1836. The numerical parameters were $\omega_{p e} d t=0.1$ and $d x=0.3 \lambda_{\text {De }}$ in the full-PIC simulations (the ions being pushed every five time steps [13]), and they were $\omega_{p i} d t=$ 0.1 and $d x=0.3 \lambda_{\mathrm{De}}$ in the hybrid simulations; here $\omega_{p e}$ and $\omega_{p i}$ denote the electron and ion plasma frequencies, $d t$ and $d x$ are the elementary time and space steps. The number of particles per cell was 200 in the hybrid simulations, and 1000 for each species in the full-PIC simulations. 
We discuss two representative cases. In the regime of weak IAW excitation, defined here as the regime where no ion wave breaking occurs [14], the normalized driver amplitude $\bar{\phi}_{d} \equiv e \phi_{d} / T_{e}$ was taken as $\bar{\phi}_{d}=0.005$, leading to a relative amplitude of IAW density fluctuation $\delta n / n_{e 0}$ which remains below the wave-breaking limit $\delta n_{\mathrm{WB}} / n_{e 0}=0.14$. In the regime of strong IAW excitation, the driver amplitude was taken as $\bar{\phi}_{d}=0.1$, and the amplitude $\delta n / n_{e 0}$ of the driven IAW was observed to rapidly reach the wave-breaking limit. The full-PIC simulations show approximately the same asymptotic IAW amplitude level for both regimes of excitation, while in the hybrid simulations, the stronger the excitation, the higher is the asymptotic level. These features are illustrated in Fig. 1, where we plot the temporal evolutions of the normalized fundamental Fourier component, $\bar{\phi}_{m} \equiv e \tilde{\phi}\left(k_{m}\right) / T_{e}$, of the potential $\phi$ associated with the driven IAW, as provided by the hybrid (dashed line) and PIC (solid line) simulations, in the weakly 1(a) and strongly 1(b) driven case. The boundary conditions were periodic, for a simulation box of length $L=8 \lambda_{m}=8 \times 2 \pi / k_{m}$.

After an initial phase of monotonic growth, the fundamental component amplitude $\bar{\phi}_{m}$ reaches its maximum $\bar{\phi}_{m, \max }$ at a time $t_{\max }$. In the weakly driven case [Fig. 1(a)], in both hybrid and full-PIC simulations, $\bar{\phi}_{m}$ decays with irregular oscillations afterwards, to end in a low asymptotic level $\bar{\phi}_{m} \simeq 1 \%-2 \%$. However, $t_{\max }$ is shorter, and the asymptotic state is reached much earlier in the full-PIC simulations than in the hybrid ones. In the strongly driven case [Fig. 1(b)], a more dramatic difference is observed between the two types of simulations: the fullPIC simulation shows a saturation level more than 10 times lower than the hybrid simulation.

We now analyze in detail the weakly driven case. We checked that the amplitude decay, taking place for times $t>t_{\max }$, is effectively due to IAW instabilities in two
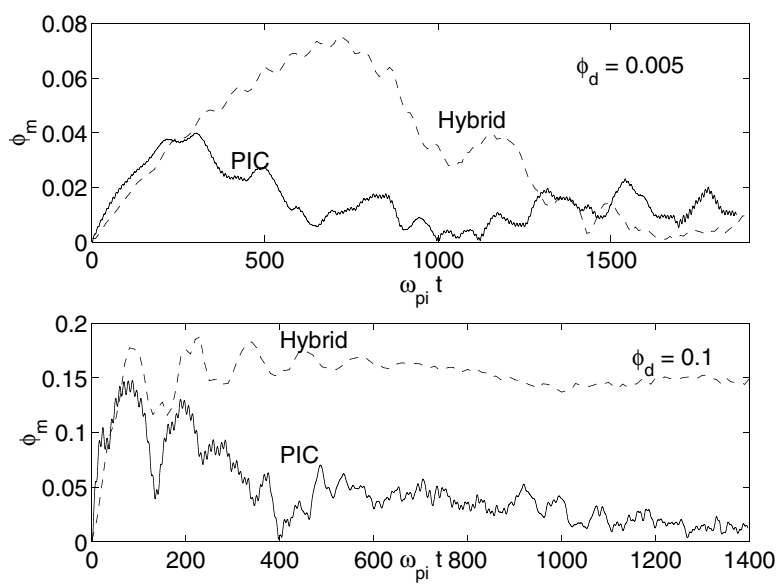

FIG. 1. The Fourier component of the fundamental IAW $\phi_{m}$ versus the dimensionless time $\omega_{p i} t$, for a driver amplitude (a) $\phi_{d}=0.005$ (weak excitation) and (b) $\phi_{d}=0.1$ (strong excitation), $k_{m}^{2} \lambda_{\mathrm{De}}^{2}=0.1$, and $T_{i} / Z T_{e}=0.09$. Solid line: fullPIC simulation; dashed line: hybrid simulation. different ways: (i) by examining the temporal evolution of the IAW $k$ spectrum, it is found that the saturation takes place at the very moment when the subharmonic content becomes broad and significant in amplitude for each type of simulation; here, the subharmonic domain in the Fourier $k$ space is defined by the inequality $|k|<k_{m}$; (ii) we carried out numerical simulations with the same physical parameters, except that we artificially quenched those IAW instabilities able to give rise to subharmonic generation by taking a short system length, namely, $L^{q}=\lambda_{m}$. In the latter simulations, the same behavior as displayed in Fig. 1(a) for $\bar{\phi}_{m_{-}}$was observed for $t<t_{\max }$, whereas the IAW amplitude $\bar{\phi}_{m}$ continues to grow for $t>t_{\max }$ until it reaches its asymptotic level in a quasimonotonic way. We may therefore assert that the temporal decay of the IAW fundamental component observed in the simulations shown in Fig. 1 is effectively due to those IAW instabilities giving rise to subharmonic generation. Therefore, our first conclusion is that the electron kinetic effects are incorrectly ignored in the hybrid description, because they actually facilitate the IAW instabilities.

As the IAW instabilities result in an energy transfer towards long wavelengths, it is interesting to investigate whether the kinetic effects might be modeled in the fluidtype equations describing the IAWs, in a way such that solving these equations would correctly reproduce the observed energy transfer. In order to test this possibility, we restricted ourselves to the initial phase of monotonic growth $t<t_{\max }$, so as to simply determine whether we would be able (i) to reproduce this initial phase within a fluid-type description, and (ii) to estimate the characteristic time for the IAW instabilities development. Concerning the initial phase of monotonic growth, a commonly used modeling of kinetic effects consists in including in the coupled mode equations the nonlinear frequency shift $\delta \omega_{\text {kin }}$ caused by the trapping of resonant particles. The equations for the evolution of $\bar{\phi}_{m}$ and of its harmonic $\bar{\phi}_{2 m}$ are then given by $[3,11]$

$$
\begin{gathered}
\left(\partial_{t}+\nu_{m}+i \delta \omega_{\mathrm{kin}}\right) \bar{\phi}_{m}=-i \omega_{m}\left[\bar{\phi}_{2 m} \bar{\phi}_{m}^{*}+\bar{\phi}_{d} / 4\right], \\
\left(\partial_{t}+\nu_{2 m}+2 i \delta \omega_{\mathrm{kin}}+i \delta \omega_{\mathrm{disp}}\right) \bar{\phi}_{2 m}=-i \omega_{m} \bar{\phi}_{m}^{2} .
\end{gathered}
$$

They are valid in the regime $\bar{\phi}_{2 m} \ll \bar{\phi}_{m}$ where the harmonic emission can be dealt with perturbatively; we checked that it is, indeed, the case by monitoring the harmonic amplitudes. We approximated the damping coefficients, $\nu_{p m}$ with $p=1$, 2, by $\nu_{p m} \simeq \nu_{p m}^{L}\left(1+t / \tau_{b i}\right)^{-1}$, so that they correspond to the linear Landau dampings $\nu_{p m}^{L}$ initially, and model their reduction due to ion trapping [15]. Here the ion bounce time, $\tau_{b i}=2 \pi / \omega_{b i}$, with $\omega_{b i} \equiv$ $\omega_{m}\left|2 \bar{\phi}_{m, \text { max }}\right|^{1 / 2}$, was estimated at the time $t_{\max }$, namely $\bar{\phi}_{m, \text { max }} \equiv \bar{\phi}_{m}\left(t_{\text {max }}\right) . \quad$ The term $\delta \omega_{\text {disp }} \equiv \omega_{L}\left(2 k_{m}\right)-$ $2 \omega_{L}\left(k_{m}\right) \simeq-3 k_{m}^{2} \lambda_{\mathrm{De}}^{2} \omega_{m}$ accounts for the IAW dispersion. In the regime of monotonic growth of the fundamental component $\bar{\phi}_{m}$, we approximated the nonlinear frequency 
shift $\delta \omega_{\text {kin }}$ by $\delta \omega_{\text {kin }}=\omega_{m} \eta\left|\bar{\phi}_{m}\right|^{1 / 2}$, with $\eta \equiv \eta_{i}+\eta_{e}$, the parameters $\eta_{s}$ corresponding to the contributions of the ions $s=i$ and electrons $s=e$. The two parameters $\eta_{s}$ have been computed in the past in some limiting cases [1517]. In order to test the relevance of the modeling (1) and to compute these two parameters, we proceeded in two ways: (i) we estimated the actual values of the parameters $\eta_{s}$ by means of a best fit, comparing the numerical simulations results with the solutions to the system (1); (ii) we checked the validity of the expression $\delta \omega_{\mathrm{kin}, s}=\omega_{m} \eta_{s}\left|\bar{\phi}_{m}\right|^{1 / 2}$ of the nonlinear frequency shift when it is caused by the nonlinearity of species $s$. To do so, we computed the nonlinear eigenfrequency $\omega_{\mathrm{NL}, s}\left(k_{m}\right)$ by solving the dispersion relation that is obtained by inserting in the susceptibility of species $s$ the spatially averaged distribution function provided by the PIC simulations carried out for the case of the short system, $L^{q}=\lambda_{m}$, and by keeping linear the susceptibility of the other species. We then determined the instantaneous parameter $\eta_{s}(t)$ by $\eta_{s}(t) \equiv \delta \omega_{\text {kin, } s} /\left(\omega_{m} \bar{\phi}_{m}^{1 / 2}\right)$, with $\delta \omega_{\text {kin, } s} \equiv \omega_{\mathrm{NL}, s}\left(k_{m}\right)-\omega_{L}\left(k_{m}\right)$. We found that past a transient stage of a few $\tau_{b i}$, the values of $\eta_{s}(t)$ are close to $\eta_{s}$ determined by the best fit.

The best fit for the hybrid simulations results was obtained for $\eta=\eta_{i}=-0.13$, and this fit appeared to be excellent. We found, on the other hand, that the instantaneous $\eta_{i}(t)$ is slightly fluctuating in time in the range $[-0.18,-0.13]$. We may thus conclude that in the case of hybrid simulations, the growth phase $t<t_{\max }$ is correctly described by the system (1), which simply models the ion kinetic effects by a nonlinear frequency shift.

In the full-PIC simulations case, the best fit was obtained for $\eta=\eta_{e}+\eta_{i} \simeq 0$, replacing $\bar{\phi}_{2 m}$ in Eq. (1a) by the value $\bar{\phi}_{2 m, \text { max }}$ observed in the simulation. Using the value $\eta_{i}=-0.13$ known from the hybrid simulation, one obtains $\eta_{e} \simeq 0.13$. Concerning the electron distribution function $f_{e}(v)$, it is slightly flattened in the thermal domain $v \approx v_{\text {the }}$, where $v_{\text {the }}$ denotes the initial electron thermal velocity; we found that it can be very well approximated by a super-Gaussian of the form $f_{e}(v) \propto$ $\exp -\left(v / \sqrt{2} \sigma v_{\text {the }}\right)^{\alpha}$, with $\sigma>1$ and $\alpha>2$ both increasing with time, indicating therefore increasing flattening with increasing $\bar{\phi}_{m}$, as expected. The flattening of the distribution function gives rise to a positive frequency shift [9] $\delta \omega_{\text {kin,e }} \sim 0.02 \omega_{m}$ at $t \sim t_{\max }$, which leads to $\eta_{e}\left(t_{\max }\right) \simeq$ 0.1 . Therefore, all these results validate the expression $\delta \omega_{\text {kin, } e}=\omega_{m} \eta_{e}\left|\bar{\phi}_{m}\right|^{1 / 2}$ together with the value of $\eta_{e} \simeq$ 0.13 . However, the second harmonic $\bar{\phi}_{2 m, \max }$ amplitude obtained from the solution of the system (1) for $\eta \simeq 0$ is found to be smaller by a factor of 4 than the value $\bar{\phi}_{2 m, \text { max }}=0.018$ observed in the simulation. Thus, although a good agreement between the best fit procedure to determine $\eta$ and the direct computation from the distribution function is observed, we must conclude that the system (1) can describe only qualitatively the initial phase dynamics in the PIC simulations.
The latter conclusion is confirmed by examining the characteristic time for the IAW instabilities development. We derived the dispersion relation associated with the stability analysis of the stationary solution $\left(\bar{\phi}_{m}, \bar{\phi}_{2 m}\right)$ to the set (1). This dispersion relation describes both the IAW instabilities associated with the fluid nonlinearity, the modulational instability driven by the nonlinear frequency shift in the case $\eta>0$, and the decay instability driven by the nonlinear frequency shift in the case $\eta<0$.

We have solved this dispersion relation numerically and derived approximate analytical expressions.

Figure 2 shows the maximum growth rate $\gamma / \omega_{p i}$ as a function of the nonlinear frequency shift $\delta \omega_{\text {kin }} / \omega_{m}=$ $\eta\left|\bar{\phi}_{m}\right|^{1 / 2}$ in the case $k_{m}^{2} \lambda_{\mathrm{De}}^{2}=0.1$, and $\bar{\phi}_{m, \max }=0.04$, characteristic of the saturation level in the full-PIC simulations. It can be seen that the IAW instability growth rate $\gamma / \omega_{p i}$ for the frequency shift $\eta\left|\bar{\phi}_{m \text {,max }}\right|^{1 / 2} \simeq 0$ is predicted to be smaller than $10^{-3}$. Therefore, the short saturation time $t_{\max } \simeq 300 \omega_{p i}^{-1}$ observed in the full-PIC simulations cannot be explained by the stability analysis associated with the system (1) modeling the kinetic effects by means of nonlinear frequency shifts. On the other hand, solving the dispersion relation for $\eta\left|\bar{\phi}_{m}\right|^{1 / 2}=-0.034$ in the case $\left(\bar{\phi}_{m, \max }=0.07, \eta=-0.13\right)$ corresponding to the hybrid simulations results, leads to the growth rate $\gamma / \omega_{p i} \simeq$ $\left|2 \delta \omega_{\mathrm{kin}} / 3 \omega_{m}\right|^{1 / 2}\left|\bar{\phi}_{m}\right| \simeq 0.01$. This prediction is in good agreement with the time $t_{\max } \simeq 700 \omega_{p i}^{-1}$ observed in the hybrid simulations, corresponding to a gain factor of a few units. These results confirm that the ion kinetic effects appear to be properly modeled by the nonlinear frequency shift, whereas the electron kinetic effects clearly cannot be reduced to a simple nonlinear frequency shift and involve another process which makes the IAW more unstable than when they are ignored.

We now comment on the strongly driven case $\bar{\phi}_{d}=0.1$. As can be seen in Fig. 1(b), the driven IAW asymptotic behavior differs significantly between the two types of simulations. In the hybrid simulation the fundamental component growth is almost monotonic, resulting in a large amplitude $\bar{\phi}_{m \text {,sat }} \simeq 0.15$. The corresponding Fourier spectrum reveals neither a significant harmonic emission nor a subharmonic content, and inspection of the ion phase space shows strong ion trapping and distortion of the

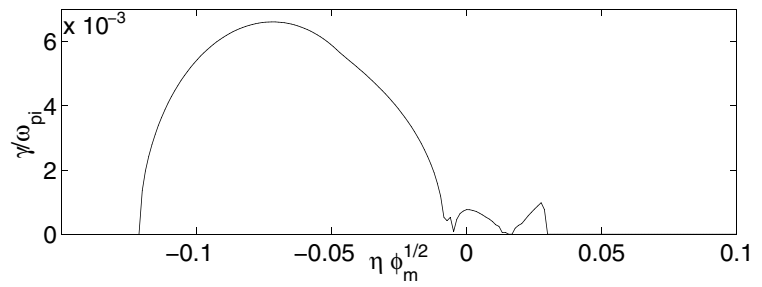

FIG. 2. Normalized growth rate $\gamma / \omega_{p i}$ obtained from the stability analysis of the equilibrium solution to Eqs. (1), for $\bar{\phi}_{m, \max }=0.04, k_{m}^{2} \lambda_{\mathrm{De}}^{2}=0.1$, versus $\delta \omega_{\mathrm{kin}} / \omega_{m}=\eta\left|\bar{\phi}_{m}\right|^{1 / 2}$. 

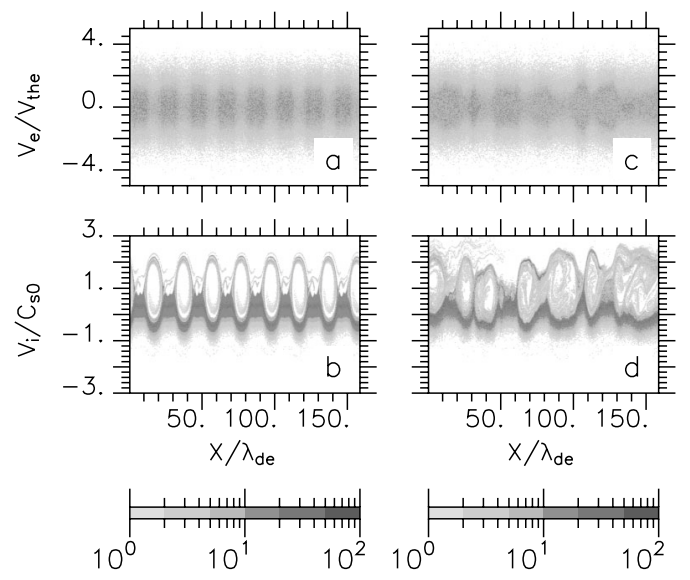

FIG. 3. (a),(c) The electron and (b),(d) ion phase spaces for the parameters of Fig. 1(b) (strongly driven case). (a),(b) Time $\omega_{p i} t=80$, and (c),(d) time $\omega_{p i} t=400$.

distribution function. All these features correspond to a Bernstein-Greene-Kruskal (mode)-like large amplitude quasimonochromatic IAW, as observed in other simulations [8].

In contrast, the full-PIC simulations lead to the same features of the IAW global behavior as those observed in the weakly driven case: after an initial growth to a large amplitude $\bar{\phi}_{m, \max } \simeq 0.15$ at a time $t_{\max } \simeq 80 \omega_{p i}^{-1}$, the fundamental component decays with irregular oscillations to end in a low amplitude level $\bar{\phi}_{m} \simeq 2 \%$. Inspection of the Fourier spectrum shows that the saturation takes place at the moment when the subharmonic content becomes significant in the spectral range $|k|<k_{m}$.

Concerning the kinetic effects, the ion phase space shows clearly the occurrence of strong ion trapping and of energy transfer into the subharmonic domain. These phenomena are illustrated in Fig. 3 corresponding to the full-PIC simulations in the strong drive case. At time $t=$ $80 \omega_{p i}^{-1}$ [Fig. 3(b)], the subharmonic content is still weak so that ion trapping is characterized by the same spatial periodicity as the driver's. At time $t=400 \omega_{p i}^{-1}$ [Fig. 3(d)], ion trapping still occurs vigorously, but it takes place in a nonperiodic way because of the significant energy transfer in the subharmonic domain. The electron phase space [Figs. 3(a) and 3(c)] shows the existence of nonlinear effects in the thermal velocity domain $|v| \leq$ $v_{\text {the }}$, with similar spatial features as for the ion phase space, and the electron distribution function is strongly flattened in the thermal velocity domain.

Concerning the interpretation of the IAW behavior in terms of the model Eqs. (1), we arrived, for the full-PIC simulations, at a still more severe conclusion than in the case of weak excitation: namely, we could not obtain any satisfactory best fit of the simulation results by modeling the electron kinetic effects in terms of a simple frequency shift, demonstrating that the electron kinetic effects interplay with the usual fluid-type nonlinearity in a nontrivial way, such that the driven IAW is more unstable than in the case when the electron kinetic effects are ignored. This interplay might correspond to an additional source term in Eq. (1b), associated with particle trapping and scaling like $\left|\bar{\phi}_{m}\right|^{3 / 2}[16]$.

In conclusion, we have demonstrated that the kinetic effects cannot be ignored when studying the nonlinear behavior of a driven ion-acoustic wave. We have also shown that the electron kinetic effects cannot be accounted for by just a nonlinear frequency shift in fluid-type equations. A complex interplay between kinetic effects and mode coupling effects leads to a turbulent state characterized by a low fluctuations level, even in the case of a strong driving. This might explain the low-level SBS saturation in laser-plasma interaction experiments. We have established this result in a collisionless one-dimensional plasma, and multidimensional and collisional effects remain to be investigated.

The authors acknowledge fruitful discussions with J.C. Adam, L. Divol, W. Rozmus, and E. A. Williams. Part of our simulations were carried out at IDRIS, Orsay (France).

[1] L. V. Powers et al., Phys. Plasmas 2, 2473 (1995); J. C. Fernandez et al., Phys. Rev. Lett. 81, 2252 (1998); S. P. Regan et al., Phys. Plasmas 6, 2072 (1999); J. D. Moody et al., Phys. Rev. Lett. 86, 2810 (2001); J. Fuchs et al., Phys. Plasmas 7, 4659 (2000); D. H. Froula et al., Phys. Rev. Lett. 88, 105003 (2002).

[2] S. J. Kartunnen, J. N. McMullin, and A. A. Offenberger, Phys. Fluids 24, 447 (1981).

[3] J. A. Heikkinen, S. J. Karttunen, and R. R. E. Salomaa, Phys. Fluids 27, 707 (1983); M. Casanova et al., Phys. Rev. Lett. 54, 2230 (1985); W. Rozmus et al., Phys. Fluids B 4, 576 (1992).

[4] S. Hüller, Phys. Fluids B 3, 3317 (1991); C. Riconda et al., Phys. Scr. T84, 217 (2000).

[5] A. V. Maximov et al., Phys. Plasmas 3, 1689 (1996).

[6] B. I. Cohen et al., Phys. Plasmas 4, 956 (1997).

[7] H. A. Rose, Phys. Plasmas 4, 437 (1997).

[8] R. E. Giacone and H. X. Vu, Phys. Plasmas 5, 1455 (1999); H. X. Vu, D.F. DuBois, and B. Bezzerides, Phys. Rev. Lett. 86, 4306 (2001).

[9] B. B. Afeyan et al., Phys. Rev. Lett. 80, 2322 (1998).

[10] L. Divol et al., Phys. Plasmas 10, 1822 (2003); E. A. Williams et al., Phys. Plasmas 11, 231 (2004).

[11] D. Pesme et al., Plasma Phys. Controlled Fusion 44, B53 (2002).

[12] P. W. Rambo, S.C. Wilks, and W. L. Kruer, Phys. Rev. Lett. 79, 83 (1997).

[13] J. C. Adam, A. Gourdin Serveniere, and A. B. Langdon, J. Comput. Phys. 47, 229 (1982).

[14] W.L. Kruer, The Physics of Laser Plasma Interaction (Addison-Wesley, New York, 1988).

[15] G. J. Morales and T. M. O’Neil, Phys. Rev. Lett. 28, 417 (1972); H. Ikezi et al., Phys. Fluids 21, 239 (1978).

[16] A. A. Andreev and V. T. Tikhonchuk, Sov. Phys. JETP 68, 1135 (1989).

[17] R. L. Dewar et al., Phys. Rev. Lett. 28, 215 (1972). 